\title{
Evaluation of Two Rapid Antigen Tests for Detection of SARS-CoV-2 Virus
}

\author{
Sahar Mohammed Khairat ${ }^{1}$, Nancy EL Guindy ${ }^{1}$, Mohammad Salah Eldeen Abdel Motaleb ${ }^{2}$, \\ Noha Salah Soliman ${ }^{3, *}$ \\ ${ }^{1}$ Department of Clinical and Chemical Pathology, Faculty of Medicine, Cairo University, Central Public Health Laboratories (CPHL), Ministry \\ of Health, Cairo, Egypt \\ ${ }^{2}$ Serology Unit, Central Public Health Laboratories (CPHL), Ministry of Health, Cairo, Egypt \\ ${ }^{3}$ Department of Clinical and Chemical Pathology, Faculty of Medicine, Cairo University, Cairo, Egypt
}

\section{Email address:}

sahar.khairat@gmail.com (S. M. Khairat), nancy_el_guindy@hotmail.com (N. EL Guindy),

Mohvirolology2014@gmail.com (M. S. E. A. Motaleb), nsal18@yahoo.com (N. S. Soliman)

${ }^{*}$ Corresponding author

\section{To cite this article:}

Sahar Mohammed Khairat, Nancy EL Guindy, Mohammad Salah Eldeen Abdel Motaleb, Noha Salah Soliman. Evaluation of Two Rapid Antigen Tests for Detection of SARS-CoV-2 Virus. International Journal of Microbiology and Biotechnology. Vol. 5, No. 3, 2020, pp. $131-134$. doi: $10.11648 /$ j.ijmb.20200503.18

Received: July 21, 2020; Accepted: August 3, 2020; Published: August 13, 2020

\begin{abstract}
Nucleic acid and antibody detection assays have been utilized in COVID-19 laboratory diagnosis. However, the use of viral antigenic proteins for diagnosis has not been successfully developed. Using viral antigen allows rapid direct viral detection earlier than production of antibodies. The present study was aimed at evaluating the performance of two COVID-19 rapid antigen detection tests, which are BIOCREDIT COVID-19 Ag (RapiGEN Inc., Korea) and Standard Q COVID-19 Ag (SD Biosensor, Korea), in comparison with RT-PCR. These tests were performed on 80 COVID-19 RT-PCR positive respiratory samples and 20 RT-PCR negative control samples. BIOCREDIT COVID-19 Ag and SD Biosensor RAD kits recorded total sensitivities of $52.5 \%$ and $68.7 \%$ and specificities of $46 \%$ and $96 \%$, respectively. In high viral load samples, BIOCREDIT COVID-19 Ag and SD Biosensor RAD kits recorded higher sensitivities of $60 \%$ and $77 \%$, compared to $45 \%$ and $60 \%$ in normal viral load samples, respectively. Sensitivity and specificity of the 2 antigen kits varied significantly with $P$ values of $<0.000001$ and 0.0135 , respectively. The evaluated RAD tests presented promising performance which was relatively better for SD-Biosensor than BIOCREDIT RAD tests, especially in high viral load samples. However, antigen tests are still considered substandard in comparison with RT-PCR in detecting SARS-CoV-2.
\end{abstract}

Keywords: COVID-19, SARS-CoV-2, Rapid Antigen Detection, RT-PCR

\section{Introduction}

COVID-19 disease is a viral respiratory infection that is caused by a novel severe acute respiratory syndrome coronavirus 2 (SARS-CoV-2), first described in China $[1,2]$. It is proved that SARS-CoV-2 is a virus that spreads rapidly with strong human-to-human transmission and was declared by the World Health Organization (WHO) as an outbreak of public health emergency that would turn into a pandemic affecting 216 countries worldwide [3]. COVID-19 infection can result in adverse complications in immunocompromised patients or patients with chronic illnesses. The clinical symptoms that are overlapping with those of seasonal flu and other respiratory infections imply accurate and confident diagnosis $[4,5]$.

Development of well-performing laboratory tests, as well as implementing effective control measures, is obligatory for diagnosing and managing COVID-19-infected patients. Due to the lack of vaccine or antiviral treatment, speedy diagnostic tests with short-time assay, which can be employed as point-of-care tests, have become a matter of great concern [6]. Rapid assays confirm COVID-19 clinical suspicion promptly, which can aid in providing early isolation and appropriate care for infected patients. Although various serological antibody 
tests have been developed, they do not fulfill the requirement of early detection of infection as the immune system takes on average 3 to 5 days to produce the antibodies [7].

Direct SARS-CoV-2 laboratory diagnosis can be achieved, through either viral nucleic acid or antigen detection in respiratory specimens. Currently, real-time reverse transcription polymerase chain reaction (RT-PCR) is the recommended molecular method for diagnosing COVID-19 acute infection. Although sensitive and specific, PCR assays are time-consuming and costly and require professional and skilled laboratory staff as well as specialized reagents and equipment. Thus, they are inconvenient when utilized on a large-scale population [8, 9].

Rapid antigen detection (RAD) tests rapidly detect certain COVID-19 viral proteins in respiratory samples and can yield results within minutes [10]. These tests are considered less expensive and faster than molecular tests. Moreover, they are more practical to be applied on a wide scale. However, antigen detection methods may suffer the same limitation that the PCR has an increased chance of false-negative results since various factors can affect the test sensitivity such as sample type, collection method, and transport conditions [11]. In order to meet the market needs, test developers were urged to rapidly introduce several laboratory COVID-19 diagnostic tests. However, this was challenged by limited chances for full performance review and process control [12]. Due to the current exceptional circumstances, an Emergency Use Authorization (EUA) policy has been issued by the Food and Drug Administration (FDA) on the $16^{\text {th }}$ of March 2020 for SARS-CoV-2 laboratory tests to $100 \mu \mathrm{L}$ be independently validated [13]. In this perspective, the current study was aimed at evaluating the performance of 2 commercially available COVID-19 RAD kits, BIOCREDIT COVID-19 Ag (RapiGEN Inc., Korea) and Standard Q COVID-19 Ag (SD Biosensor, Korea), compared to gold standard RT-PCR in terms of sensitivity, specificity, and accuracy.

\section{Methods}

\subsection{Sample Collection and Study Population}

A total number of hundred nasopharyngeal (NP) specimens were utilized in the present study. The hundred specimens were in the form of 80 samples collected from real-time polymerase chain reaction (RT-PCR) COVID-19 positive patients, which were received at the Virology Department of Central Public Health Laboratories (CPHL), Ministry of Health in Egypt, and twenty samples obtained from normal healthy individuals tested negative for RT-PCR-COVID-19 as control group for test specificity. The eighty RT-PCR COVID-19 positive samples were further categorized into 40 high viral load and 40 normal viral load samples.

\subsection{COVID-19 Rapid Antigenic Detection (RAD) Tests}

All samples were tested for SARS-CoV-2 antigens using two commercially available rapid antigen detection (RAD) kits in Egypt: BIOCREDIT COVID-19 Ag (RapiGEN Inc.,
Korea) and Standard Q COVID-19 Ag (SD Biosensor, Korea) that were provided to the CPHL in order to assess the performance of these tests before introduction to routine.

COVID-19 RAD kits are rapid and qualitative tests that allow detecting SARS-CoV-2 antigen in nasopharyngeal specimens. These tests utilize monoclonal antibodies conjugated to colloidal gold nanoparticles in order to target SARS-CoV-2 antigen. These antibodies are fixed onto nitrocellulose membrane. Antigen detection was conducted as per the manufacturers' instructions through mixing $100 \mu \mathrm{L}$ of nasopharyngeal secretions with 4 drops of dilution buffer in a tube, which is then added to the strip, where solubilized conjugate together with the sample flows with passive diffusion and reacts with SARS-CoV-2 antibodies fixed onto the nitrocellulose membrane. The strip includes a control line in order to ensure correct flow of the sample. The results were interpreted visually after 15 minutes [5]. Sensitivity, specificity, and accuracy were considered when evaluating the performance of both kits, in comparison with the gold standard viral nucleic acid detection using RT-PCR.

\subsection{Performance Evaluation and Statistical Analysis}

We evaluated the performance of these tests as per the guidelines of the Clinical and Laboratory Standards Institute [14]. Sensitivity, specificity, and accuracy were considered when evaluating each of the tests. Sensitivity represents how frequently the antigen test yields true positive results for truly affected patients. Specificity measures the proportion of how frequently the antigen test is negative when the COVID-19-free volunteer is accurately identified as PCR negative for COVID-19. Binomial 95\% confidence intervals (CI) were calculated for proportions. Cohen Kappa index was calculated between both test kits. We compared the differences in performance of both kits using Chi-square $\left(\mathrm{X}^{2}\right)$ test. $P$ value less than 0.05 was considered statistically significant. Microsoft Excel 2010 (Microsoft Corp., Redmond, WA) and SPSS (Statistical Package for the Social Science; SPSS Inc., Chicago, IL, USA) version 15 for Microsoft Windows (Microsoft Corp., Redmond, WA) were utilized for conducting all statistical analyses.

\subsection{Ethical Statement}

This research received an institutional approval and was conducted upon a request from the Central Public Health Laboratories (CPHL) of the Ministry of Health, Egypt, in order to assess the performance of 2 commercial COVID-19 rapid antigen detection kits. No clinical trials or invasive procedures or laboratory animals were involved in this study.

\section{Results}

The present study enrolled 100 respiratory samples which were classified into 80 RT-PCR COVID-19 positive samples and 20 RT-PCR-COVID-19 negative NP specimens as control group. The eighty RT-PCR-COVID-19 positive respiratory samples were classified into 40 high viral load samples $(\mathrm{Ct}$ 
values <18.57) and 40 normal viral load samples ( $\mathrm{Ct}$ values $>18.57$ ).

As presented in Table 1, the total sensitivity for BIOCREDIT COVID-19 Ag and SD Biosensor RAD kits was found to be $52.5 \%$ (95\% CI: $0.68-0.91)$ and $68.7 \%$ (95\% CI: 0.62-0.83), respectively. In high viral load samples, the sensitivity for BIOCREDIT COVID-19 Ag and SD Biosensor RAD kits was 60\% (95\% CI: 0.54-0.86) and 77\% (95\% CI:
0.47-0.75), while in normal viral load samples, it was $45 \%(95 \%$ CI: $0.46-0.82)$ and $60 \%$ (95\% CI: 0.40-0.70), respectively. The recorded specificity was $46 \%$ (95\% CI: $0.14-0.41$ ) and $96 \%$ (95\% CI: 0.002-0.28) for BIOCREDIT COVID-19 Ag and SD Biosensor, respectively. The 2 antigen kits varied significantly in sensitivity and specificity with $P$ values of $<0.000001$ and 0.0135 , respectively. The 2 antigen kits recorded fair agreement of $77 \%$ (kappa: 0.22 ).

Table 1. Results of COVID-19 RAD tests' analytical sensitivity, specificity, and accuracy.

\begin{tabular}{|c|c|c|c|c|c|}
\hline COVID-19 RAD tests & Sensitivity\% (95\% CI) & NPV & Specificity\% (95\% CI) & PPV\% & Accuracy \% \\
\hline BIOCREDIT COVID-19 Ag test (RapiGEN) & $52.5(0.68-0.91)$ & 21.9 & $45(0.14-0.41)$ & 81.3 & 51 \\
\hline Standard Q COVID-19 Ag (SD Biosensor) & 68.7 & 43.1 & $95(0.002-0.28)$ & 98.4 & 74 \\
\hline
\end{tabular}

PPV: Positive Predictive Value, NPV: Negative Predictive Value, 95\% CI: 95\% Confidence Interval, and RAD: Rapid Antigen Detection.

\section{Discussion}

COVID-19 diagnostic testing plays a vital role in managing SARS-CoV-2 infected patients appropriately and controlling the spread of the virus. Currently, molecular and immunoassays are the most commonly known diagnostic categories related to COVID-19 that overlap in the COVID-19 pandemic state [8]. Several diagnostic tests have been developed in order to detect SARS-CoV-2 viral antigens in respiratory samples. These tests have been independently validated by test developers and rapidly introduced to the market. The current study evaluated the performance of 2 commercially available COVID-19 RAD tests. SD Biosensor had better performance than BIOCREDIT antigen tests showing significantly higher sensitivity (68.7\% vs. $52.5 \%$, respectively) with a $P$ value $<0.0000001$ and higher specificity (95\% vs. $45 \%$, respectively) with a $P$ value $<0.0135$. To date, published data regarding the performance of COVID-19 antigen tests are still limited. However, performance data concerning various SARS-CoV-2 diagnostic methods from different laboratories worldwide are shared on an electronic platform by the Foundation of Innovative New Diagnostics (FIND), a nonprofit global organization [15]. According to FIND, the evaluation of BIOCREDIT antigen test showed higher sensitivity (62\%) and specificity $(100 \%)$ in comparison with our study. Few studies evaluated the performance of various COVID-19 RAD tests but for manufacturers other than those presented in our study. Among these studies, Mak et al. [16] conducted a study in which they recorded a total sensitivity of $30.2 \%$ for Resp strip COVID-19 antigen test. Mertens et al. [5] conducted another study in which they evaluated the performance of Resp strip COVID-19 antigen test and reported sensitivity and specificity of $57.6 \%$ and $99.5 \%$, respectively. In the current study, the sensitivity of BIOCREDIT and SD Biosensor was observed to be higher in high viral load RT-PCR positive samples (Ct values <18.57) $(60 \%, 77.5 \%)$ compared to low viral load samples ( $\mathrm{Ct}$ values $>18.57)(45 \%, 60 \%)$. This observation agreed with that of Scohy et al. [10] who reported that COVID-19 antigen tests show high sensitivity in high viral load samples $(\mathrm{Ct}$ values $<25)$ which declines when viral load decreases (Ct values $>30$ ). Likewise, Mertens et al. [5] demonstrated that higher sensitivity of $74.2 \%$ was observed in the subpopulation with high viral load $(\mathrm{Ct}$ values $<25)$ of SARS-CoV-2. Being rapid, easy to use, and of low cost with no demand for special equipment of skilled personnel are of the main advantages of COVID-19 RAD tests; thus, these tests are more preferable than PCR, especially when applied on a wide scale [10]. However, the analytical performance of COVID-19 RAD tests depends on several factors related to specimen quality, processing, and inhered viral load. Similar to many of the developed commercially available lateral flow immunoassays, COVID-19 rapid antigen detection tests have challenging sensitivity in comparison with molecular assays [5]. Furthermore, although COVID-19 RAD tests can detect actively infected patients, they cannot identify people who had been previously infected or developed immunity [9]. This is also one limitation of molecular assays as they cannot identify patients with past infection.

To the best of our knowledge, this is the first study from Egypt evaluating the 2 currently available COVID-19 rapid antigen detection tests in the market in order to provide potential guidance about their analytical performance. Further researches are needed to enrich the field with evidence-based data and to offer guidance for well-performing commercially available COVID-19 antigen tests in the market.

\section{Conclusion}

Either COVID-19 nucleic acid detection by PCR or antigen detection tests can be utilized in respiratory samples for direct detection of SARS-CoV-2. The overall performance of the 2 evaluated RAD tests was promising. SD Biosensor showed relatively better performance than BIOCREDIT. Higher sensitivities were recorded in high viral load samples compared to normal ones. However, so far antigen tests do not yet live up to RT-PCR in SARS-CoV-2 detection.

\section{References}

[1] Zhu N, Zhang D, Wang W, Li X, Yang B, Song J, et al. (2019). A novel Coronavirus from patients with Pneumonia in China. $\mathrm{N}$ Engl J Med, 382: 727-33. 10.1056/NEJMoa2001017. 
[2] Huang C, Wang Y, Li X, et al. (2020). Clinical features of patients infected with 2019 novel coronavirus in Wuhan, China. Lancet, $395 \quad$ (10223): 497-506, doi: https://doi.org/10.1016/S0140-6736 (20)30183-5.

[3] World Health Organization (WHO) (2020). [Accessed 15 April 2020]

https://www.who.int/emergencies/diseases/novel-coronavirus2019/global-research-on-novel-coronavirus-2019-ncov.

[4] Xiang F, Xiaorong W, Xinliang H, Zhenghong P, Bohan Y, Jianchu Z, et al. (2020). Antibody detection and dynamic characteristics in patients with COVID-19. Clinical Infectious Diseases: An Official Publication of the Infectious Diseases Society of America, https://doi.org/10.1093/cid/ciaa461.

[5] Mertens P, De Vos N, Martiny D, Jassoy C, Mirazimi A, Cuypers L et al. (2020). Development and potential usefulness of the COVID-19 Ag Respi-Strip diagnostic assay in a pandemic context. Front Med (Lausanne), 7: 225. doi: 10.3389/fmed.2020.00225.

[6] Rubin EJ, Baden LR, Morrissey S. (2020). Audio interview: new research on possible treatments for Covid-19. N Engl J Med, 382: e30. 10.1056/NEJMe2005759.

[7] Shi Y, Wang Y, Shao C, Huang J, Gan J, Huang X, et al. (2020). COVID-19 infection: the perspectives on immune responses. Cell Death Differ, 27: 1451-54. 10.1038/s41418-020-0530-3.

[8] Carter L, Linda VG, Jeffrey WS, Yingzhu L, Qiongqiong Z, Catherine JS, Janet MS, et al. Assay Techniques and Test Development for COVID-19 Diagnosis. ACS Central Science 2020, https://doi.org/10.1021/acscentsci.0c00501.

[9] Tahamtan A, Abdollah A. (2020). Real-Time RT-PCR in COVID-19 detection: issues affecting the results. Expert
Review of Molecular Diagnostic, 20 (5): 453-454, https://doi.org/10.1080/14737159.2020.1757437.

[10] Scohy A, Anantharajah A, Bodéus M, Kabamba-Mukadi B, Verroken A and Rodriguez-Villalobos H. (2020). Low performance of rapid antigen detection test as frontline testing for COVID-19 diagnosis. J Clin Virol, 129: 104455.

[11] Udugama B, Kadhiresan P, Kozlowski HN, Malekjahani A, Osborne M, Li VYC, et al. (2020) Diagnosing COVID-19: the disease and tools for detection. ACS Nano, 14 (4): 3822-3835, https://doi.org/10.1021/acsnano.0c02624 14, 3822.

[12] Cassaniti I, Novazzi F, Giardina F, et al. (2020). Performance of VivaDiag COVID-19 IgM/IgG Rapid Test is inadequate for diagnosis of COVID-19 in acute patients referring to emergency room department. J Med Virol, 10.1002/jmv.25800. doi: $10.1002 / j m v .25800$.

[13] Food and Drug Administration (FDA). (2020). [Accessed 24 April 2020]

https://www.fda.gov/medical-devices/emergency-situations-m edical-devices/faqs-testing-sars-cov-2.

[14] Clinical and Laboratory Standards Institute (CLSI). (2008). User Protocol for Evaluation of Qualitative Test Performance; Approved Guideline-Second Edition. CLSI document EP12-A2. Wayne, PA: Clinical and Laboratory Standard Institute.

[15] Foundation of Innovative New Diagnostics (FIND). (2020). [Accessed 24 April 2020] https://www.finddx.org/covid-19/dx-data/.

[16] Mak GCK, Cheng PKC, Lau SSY, Wong Kky, Lau CS, Lam ETK et al. (2020). Evaluation of rapid antigen test for detection of SARS-CoV-2 virus. J Clin Virol, 129: 104500. 